\title{
PROMOVE - CRIANÇAS: AVALIANDO EFEITOS DO PROTOCOLO EM CRIANÇAS COM PROBLEMAS DE COMPORTAMENTO
}

\section{PROMOTE - CHILDREN: EVALUATING EFFECTS OF THE PROTOCOL IN CHILDREN WITH BEHAVIOR PROBLEMS}

Tâmily Miott ${ }^{1}$

Alana Gabriela Polesello

Thais Cristina Gutstein Nazar ${ }^{3}$

\section{RESUMO}

O objetivo deste trabalho foi verificar os efeitos da aplicação do protocolo PROMOVE-Crianças (Bolsoni-Silva \& Falcão, 2016) para problemas de comportamento na infância. Avaliaram-se os repertórios comportamentais das crianças participantes, por meio da aplicação dos testes Child Behavior Checklist (CBCL) e dos estudos parentais com o Inventário de Estilos Parentais (IEP). Quando práticas desfavoráveis estão presentes no repertório comportamental, a possibilidade do surgimento de déficits de habilidades sociais e de problemas de competência social aumentam de forma significativa, impactando negativamente nas relações interpessoais e interferindo diretamente na qualidade de vida das crianças e adolescentes (Bolsoni-Silva \& Falcão, 2016). Identificaram-se no presente estudo a predominância de práticas parentais educativas negativas, estilos parentais de risco em 71,42\% dos participantes, 14,29\% apresentaram estilo parental regular abaixo do esperado e 14,29\% com estilo parental regular acima do esperado. Com relação ao comportamento, verificou-se que todos os resultados estão acima da média da padronização do instrumento, sinalizando problemas de comportamentos, tanto antes quanto após a aplicação do Protocolo. Contudo, observou-se a diminuição no escore do Pós-teste em relação ao Pré-teste. Sendo assim, é fundamental o estudo científico do desenvolvimento psicossocial, bem como o domínio das práticas educativas positivas e comportamentos habilidosos, proporcionando qualidade nas interações sociais das crianças e familiares.

Palavras-chave: Comportamento infantil, Habilidades Sociais, Estilo Parental.

1 Graduanda do curso de Psicologia pela Universidade Paranaense - Unipar. Francisco Beltrão-PR, Brasil. E-mail: tamilymiott@hotmail.com

2 Graduanda do curso de Psicologia pela Universidade Paranaense - Unipar. Francisco Beltrão-PR, Brasil. E-mail: alanapolesello@outlook.com

3 Doutora em Educação pela Universidade Federal do Paraná (UFPR). Professora no Curso de Psicologia da Universidade Paranaense - UNIPAR.E-mail: thaiscg@prof.unipar.br 


\section{ABSTRACT}

This study had as objective to verify the effects of the PROMOVE - Children (Bolsoni-Silva \& Falcão, 2016) protocol for behavioral problems in childhood. The behavioral repertoires of the participanting children by applyng the Child Behavior Checklist (CBCL) tests and parental studies with the Parenting Styles Inventory (IEP) were evaluated. When unfavorable practices are presente in the behavioral repertoire, the possibility of the emergence of social skills déficits and problems of increased social competence, negatively impacting interpersonal relationships and directly interfering in the quality of life of children and (Bolsoni - Silva \& Falcão, 2016). In the present study the predominance of negative educational parenting practices, risky parenting styles in $71,42 \%$ of participants, $14,29 \%$ with below - expected regular parenting style and $14,29 \%$ with above - expected regular parenting style were identified. Regarding behavior, verify that all results are above the averege of the standardization of the instrument, signaling behavior problems, both before and after protocol application. However, it was observed the decrease in post - test score compared to the pre - test. Thus, the scientific study of psychosocial development, as well as the positive educational practices mastery, and skillful behaviors, providing quality in the social interactions of children and their families, is fundamental.

Keywords: Child Behavior. Social Skills. Parenting Style.

\section{INTRODUÇÃO}

O desenvolvimento de habilidades, de acordo com Farias, Fonseca \& Nery (2018), ocorre por conta de contingências de comportamentos filogenéticos, ontogenéticos e culturais, influenciando os relacionamentos interpessoais na infância. O quesito filogenético diz respeito à seleção de comportamentos inatos ao longo da história evolucionária da espécie, enquanto que o nível ontogenético se refere à seleção de comportamentos durante a história de vida de um organismo. Além desses, o nível cultural trata da seleção de práticas culturais ao longo da história de uma cultura. A partir dessa descrição, os autores apontam que "[...] não há um evento único ou uma causa que produza linear e diretamente um efeito, mas, sim, relações funcionais, de maneira que o comportamento é considerado uma variável dependente em relação aos eventos ambientais, os quais seriam variáveis independentes" (Farias, Fonseca \& Nery, 2018, p. 23).

A aquisição de habilidades sociais e o desenvolvimento de competência social constituem processos que acontecem de modo natural, por meio de interações cotidianas. De acordo com Del Prette e Del Prette (2017), as práticas educativas utilizadas pela família e pela escola, somadas ao convívio com os colegas, tornam-se as principais contingências para a aquisição e para o aperfeiçoamento de comportamentos habilidosos ou não habilidosos adquiridos no repertório comportamental da criança. 
Desse modo, pode-se considerar que um repertório de habilidades sociais, como a déficits em comportamentos como empatia, expressão de sentimentos e resolução de problemas depende de fatores cognitivos e emocionais como a baixa autoestima, o baixo autoconceito, as crenças e as atribuições disfuncionais, a impulsividade e o temperamento difícil, entre outros (Cia \& Barham, 2009).

De acordo com Portella (2011, p. 17), “[...] não existe uma única definição para o termo habilidade social, assim como não existe consenso quanto às dimensões comportamentais das HS." Para tanto, podemos considerar habilidades sociais como um repertório de "[...] comportamentos que podem expressar atitudes, desejos, opiniões ou direitos de uma forma adequada à situação, respeitando a expressão desses comportamentos nos outros" (Knight, 2000 conforme citado por Del Prette \& Del Prette, 2017, p. 176). Além disso, Portella (2011, p. 18) explica que

Habilidade social é a denominação dada às diferentes classes de comportamentos sociais disponíveis no repertório de uma pessoa, que contribuem para a qualidade e a efetividade das interações que ela estabelece com as demais. Tais habilidades dizem respeito a comportamentos necessários a uma relação interpessoal bem-sucedida, conforme parâmetros típicos de cada contexto e cultura [...].

Já o Treinamento de Habilidades Sociais (THS, doravante), segundo Del Prette e Del Prette (2017), é um procedimento pelo qual se aprende habilidades sociais e pode ocorrer de modo incidental ou de modo planejado, por meio de ensino ou de psicoterapias. Além disso, devemos considerar que o contexto social tem grande influência, pois a valorização das habilidades sociais varia de acordo com o contexto social e cultural que o sujeito está inserido.

Desse modo, quando práticas educativas desfavoráveis tais como agressividade, dificuldade em conversar e impor regras, até mesmo superproteção estão presentes no repertório comportamental, a possibilidade do surgimento de déficits em habilidades sociais e de problemas de competência social aumenta de forma significativa, impactando negativamente as relações interpessoais e interferindo diretamente na qualidade de vida das crianças e adolescentes (Bolsoni-Silva \& Falcão, 2016). Os comportamentos que decorrem de problemas que causam prejuízos no seu funcionamento social geralmente se iniciam em casa, quando a criança apresenta estímulos aversivos contingentes ao comportamento de outras pessoas da família (Weber, 2008). Assim, podemos compreender que a criança apresentará dificuldades no desenvolvimento de habilidades sociais, assim como na aprendizagem.

Tendo em vista as dificuldades de alguns pais na educação de seus filhos, é imprescindível o apoio social, haja vista que, segundo Bee e Boyd (2011), os pais que têm apoio emocional e físico adequado são capazes de responder aos seus filhos com mais afeto, consistência e controle, permitindo melhores habilidades de paternagem. De acordo com Gomide (2014), o estilo parental é definido como um conjunto de práticas educativas parentais ou de atitudes parentais utilizados pelos cuidadores com o objetivo de educar, socializar e controlar o comportamento dos filhos. Isso quer dizer que pais que apresentam dificuldades em 
seguir regras e normas sociais, precisam assimilar que o sucesso de suas ações dependerá do estabelecimento de uma relação familiar que forneça aceitação emocional, moderação, supervisão e modelagem de comportamentos.

Os autores Del Prette e Del Prette (2008) apontam que, quando o repertório de habilidades sociais na família é defasado, o processo de aprendizagem é afetado de forma que o rendimento apresenta-se menor do que o esperado, considerando fatores como a idade e a série escolar. Entre essas atividades, podemos avaliar a leitura, a escrita, a fala e o raciocínio que influenciarão para além do contexto escolar, atingindo diversos aspectos na vida da criança.

As habilidades não desenvolvidas como dificuldade em expressar direitos e necessidades adequadamente, esperar sua vez, admitir erros, pedir desculpas e ouvir críticas, estão intrinsecamente relacionadas à história de vida e ao repertório comportamental das crianças que apresentam dificuldades no processo de aprendizagem. Por isso, é fundamental a promoção de habilidades sociais, gerando nelas aumento do repertório comportamental, em que habilidades serão moldadas para que o expressar-se torne-se algo natural, desencadeando, assim, significativo desenvolvimento no processo de leitura e de escrita, além de progresso com relação aos comportamentos sociais.

$\mathrm{Na}$ terapia grupal, é possível identificar, avaliar e intervir diretamente nos problemas de comportamento, desenvolvendo novas formas de interação, preparando as crianças para agirem da mesma forma em ambientes fora da clínica. Como indica Ferraz (2005), o trabalho grupal possibilita que o terapeuta identifique o comportamento-problema, assim como, atue sobre ele utilizando estratégias que envolvam as crianças na própria situação.

Em grande parte dos programas que desenvolvem habilidades sociais em grupo, utilizamse atividades lúdicas, técnicas analítico-comportamentais, além do ensaio comportamental, da modelação e da modelagem, empregando também atividades de casa, para que o sujeito amplie o seu repertório para além do contexto do programa (Del Prette \& Del Prette, 2017).

De acordo Del Prette e Del Prette (1996), a partir dos anos de 1980, os programas de THS passaram a ser destinados com maior frequência a uma clientela não clínica, buscando por padrões mais satisfatórios de vida nas comunidades em que os sujeitos estão inseridos, como a família, a escola e o grupo de amigos. Esses programas surgem nos settings terapêuticos individuais e movimentam-se para as intervenções em grupo, nos quais o trabalho se mostrou mais efetivo, já que os conhecimentos dos membros podem ser compartilhados com os demais, favorecendo trocas de experiências, opiniões, sentimentos, entre outros (Bolsoni-Silva, 2002).

Podemos citar o exemplo do programa de THS desenvolvido por Salvom Mazzarotto e Löhr (2005). Juntos à equipe da Universidade Federal do Paraná, formaram um grupo de crianças com idade entre quatro e seis anos e que integra o atendimento aos pais. A intervenção consistiu-se em 10 encontros semanais. O grupo continha em média seis crianças. Tanto para as crianças quanto para os pais, os temas trabalhados foram solução de problemas, a leitura do contexto em que estavam inseridos, a assertividade e a empatia. Contudo, no grupo de crianças, foram feitas atividades lúdicas, enquanto o grupo de pais recebeu instruções acerca de como prover condições antecedentes e reforçadoras de comportamentos socialmente habilidosos em seus filhos. Orientações semelhantes às fornecidas aos pais foram também trabalhadas com 
os professores das crianças participantes do programa, sendo esses encontros ocorriam a cada 15 dias. Uma sessão individual para devolução dos resultados aos pais e para outros encaminhamentos foi feita ao fim da intervenção. Foram avaliados os resultados por meio da observação direta do comportamento das crianças, feitas durante atividades escolares e durante a sessão terapêutica e de respostas dos pais ao Child Behavior Check-list de Achenbach (CBCL), antes e após a intervenção. Os relatos dos pais mostraram ampliação no repertório de habilidades sociais das crianças; todavia, não confirmados por meio dos dados observacionais obtidos durante as atividades escolares e as sessões terapêuticas. Dessa forma, foram discutidas sugestões para aprimoramento dos procedimentos de avaliação e de cuidados para aumentar a adesão ao programa interventivo. Em função das desistências ocorridas ao longo dos encontros, as autoras advertiram que os resultados não foram generalizados, pois apenas pais altamente motivados permaneceram até o fim da intervenção.

A principal motivação para sustentar o presente estudo reside na importância que os problemas de comportamento em crianças têm para a Psicologia, principalmente por se tratar de crianças em fase de desenvolvimento, sendo fundamental o estudo científico do desenvolvimento psicossocial no que se refere às habilidades sociais na infância. Como cita Papalia (2013, p. 36), “o campo do desenvolvimento humano concentra-se no estudo científico dos processos sistemáticos de mudança e estabilidade que ocorrem nas pessoas”.

Diante desse panorama, o objetivo deste trabalho foi verificar os efeitos da aplicação do protocolo PROMOVE - Crianças (Bolsoni-Silva \& Falcão, 2016), bem como aferir as mudanças de comportamento nas crianças participantes por meio da aplicação dos testes Child Behavior Checklist (CBCL) e Inventário de Estilos Parentais (IEP, doravante), considerando que o desenvolvimento da sociabilidade humana está envolto em um conjunto de transformações que acontecem por toda a extensão do ciclo vital, qualificando as relações e as interações com as outras pessoas e, ao mesmo tempo, nos processos cognitivos e afetivos.

\section{Método}

O estudo foi desenvolvido por meio da utilização de 10 sessões do THS com crianças de 9 a 12 anos, encaminhados pelo serviço-escola do Centro de Psicologia Aplicada (CPA) de uma universidade no estado do Paraná, em lista de espera.

Todas as ações tinham como intuito fortalecer o repertório de comportamentos que contribuem para que as crianças reduzam problemas no contexto escolar e familiar. Para isso, o processo interventivo com as crianças teve como objetivo modelar seus repertórios, inserindo comportamentos que sejam propícios a interações sociais, desenvolvendo percepções acerca da importância das relações, possibilitando um clima positivo em sala de aula, nas interações com os colegas e familiares, e melhorando a discriminação e o autocontrole das emoções. 


\section{Participantes}

A presente pesquisa foi aprovada pelo Comitê de Ética em Pesquisa da Universidade Paranaense (UNIPAR), conforme o Parecer: 3.146.331 e no CAAE: 93703418.4.0000.0109, e se pauta nos princípios e nas normas estabelecidas pela Resolução 466/2012.

Participaram do estudo sete crianças regularmente matriculadas em escolas públicas de Ensino Fundamental, no município de Francisco Beltrão, estado do Paraná. Esses participantes foram todos do gênero masculino, considerando que não haviam crianças do gênero feminino em lista de espera com queixas que pudessem ser trabalhadas durante a aplicação do projeto.

Os participantes tinham idade entre 9 e 12 doze anos, sendo que dois tinham nove anos, um tinha 10 anos, três tinham 11 anos e um tinha 12 anos. Conforme indica a Tabela 1, todos frequentavam o quarto, quinto, o sexto e sétimo anos, em escolas públicas do município. Sobre dados familiares, dois dos participantes moravam com a mãe, uma criança morava com o pai e com a mãe, outro morava com o pai, a mãe e irmãos, outro morava somente com o pai, um morava com a mãe e irmãos e apenas uma criança morava em Casa Abrigo.

Todas as crianças encontravam-se em fila de espera para atendimento psicoterapêutico, no CPA. A busca por atendimento ocorreu em maior parte por encaminhamento realizado pelas respectivas escolas, por procura espontânea de seus pais e uma das crianças foi direcionada pela Casa Abrigo. Os critérios utilizados para inclusão ao grupo foram a idade das crianças, bem como as dificuldades relatadas como queixa. A maioria dos participantes tinham queixas semelhantes e parte deles apresentou duas ou mais queixas, conforme a Tabela 1. Entre as queixas, alistam-se: dificuldades de aprendizagem, dificuldades no relacionamento familiar e dificuldades no relacionamento social e dificuldades em controlar as necessidades fisiológicas.

Também participaram indiretamente deste estudo os pais, cuidadores e/ou responsáveis civis das crianças, sendo que, primeiramente, aceitaram e permitiram a participação das crianças, realizaram uma entrevista inicial e responderam aos instrumentos sobre os comportamentos das crianças e dos estilos parentais antes e após o início das intervenções com crianças.

Tabela 1

Descrição dos participantes conforme Idade, Série, Queixa, Responsável

\begin{tabular}{l|r|l|l|l}
\hline Participante & \multicolumn{1}{|c|}{ Idade } & \multicolumn{1}{|c|}{ Ano } & \multicolumn{1}{|c}{ Queixa } & Responsável \\
\hline P1 & 9 & Quinto Ano & Dificuldade de Aprendizagem Acadêmica & Mãe \\
\hline P2 & 9 & Quarto Ano & $\begin{array}{l}\text { Dificuldade em Controlar as necessidades } \\
\text { fisiológicas }\end{array}$ & Pai \\
\hline P3 & 11 & Sétimo Ano & Dificuldade de Aprendizagem Acadêmica & Mãe \\
\hline P4 & 11 & Sexto Ano & Dois ou mais dos itens* & Mãe Social \\
\hline P5 & 10 & Sexto Ano & Dois ou mais dos itens* & Pai \\
\hline P6 & 11 & Sexto Ano & Dois ou mais dos itens* & Mãe \\
\hline P7 & 12 & Sétimo Ano & Dois ou mais dos itens* & Mãe \\
\hline Pota Apresen
\end{tabular}

Nota. Apresentação de dificuldade de aprendizagem, relacionamento familiar, relacionamento social e dificuldades em controlar as necessidades fisiológicas. 


\section{Local}

O presente estudo foi desenvolvido no CPA, espaço construído e privado para uso dos acadêmicos de Psicologia. Constitui-se como um importante projeto de extensão que atende à comunidade de Francisco Beltrão e região, oferecendo suporte psicológico tanto individual, familiar ou em grupo, e às instituições do município, proporcionando o exercício da profissão do psicólogo na área clínica e na prevenção e promoção da saúde. $O$ CPA foi incorporado à Universidade Paranaense (UNIPAR), agindo em diversas esferas de nossa comunidade, cumprindo o seu papel social e tendo como objetivo atender a toda a comunidade, sendo crianças, adolescentes, adultos e a melhor idade, na busca da saúde mental e possibilitando ao usuário conhecer melhor a si mesmo a partir da sua relação com o outro.

\section{Instrumentos}

a) Child Behavior Checklist - CBCL - (Achenbach \& Rescorla, 2001): avalia a competência social de crianças e de adolescentes por faixa etária. Existem duas versões: uma para crianças de um ano e meio a cinco anos e outra para crianças e adolescentes de seis a 18 anos. Em ambos os instrumentos, o informante deverá ser os pais ou cuidadores. É composto de 99 sentenças que se referem a problemas de comportamento e à competência social. A escala é composta por 20 itens que incluem atividades da criança, como brincadeiras, jogos e tarefa, participação em grupos, os quais deverão ser avaliados pelo respondente como não verdadeira - tanto quanto se sabe; um pouco verdadeira ou algumas vezes verdadeira; ou muito verdadeira ou frequentemente verdadeira, o que corresponde, respectivamente, a 0, 1 e 2 pontos na escala (Gallo, Grossi \& Wielewicki, 2011,). Tal instrumento foi utilizado para avaliar o funcionamento comportamental, social e emocional das crianças.

b) Inventário de Estilos Parentais - IEP (Gomide, 2014): tem por "objetivo estudar a maneira utilizada pelos pais na educação de seus filhos" (p. 77). O teste é composto por 42 questões, sendo essas direcionadas a sete variáveis, sendo elas: monitoria positiva; comportamento moral; punição inconsistente; negligência; disciplina relaxada; monitoria negativa; abuso físico. A partir da avaliação dos testes respondidos pelos responsáveis legais, pode-se classificar o estilo parental em quatro classes: ótimo, regular acima da média, regular abaixo da média e de risco. $\mathrm{O}$ instrumento foi utilizado com objetivo de avaliar as práticas educativas parentais presentes nas famílias de cada participante, pois elas podem afetar o desenvolvimento das crianças, haja vista que as práticas aplicadas têm grande influência no processo interventivo, ou seja, podem auxiliar ou dificultar o processo. 
c) Protocolo PROMOVE - Crianças, Treinamento de Habilidades Sociais (BolsoniSilva \& Falcão, 2016): é um instrumento composto por roteiros de 10 sessões, as quais apresentam procedimentos que contemplam habilidades sociais necessárias às crianças com problemas de comportamento, ampliando seu repertório de habilidades sociais, promovendo melhores nas interações e prevenindo problemas de comportamento por meio de atividades práticas e reflexivas de maneira que estimulam as crianças a produzirem reflexões sobre seus comportamentos. Foi utilizado na tentativa de ampliar o repertório das crianças, a partir de diversos recursos terapêuticos, possibilitando um entendimento mais acessível, assim como momentos de reflexão em que era possível relacionar vivências pessoais com a habilidade social.

\section{Procedimentos}

Conforme a Tabela 2, durante o primeiro encontro, as crianças foram acolhidas e conheceu-se um pouco de cada uma; na sequência, foi estabelecido o contrato. Foram realizadas 10 sessões com as crianças, aplicando atividades de intervenção, sendo que em cada encontro foram abordados diferentes assuntos relacionados às habilidades sociais (Bolsoni-Silva \& Falcão, 2016).

De acordo com a proposta, foram trabalhados temas como cumprimentar e iniciar conversas de civilidade, agradecer, falar coisas boas e expressar opiniões, fazer amigos e ajudar, brincar e dividir as coisas, esperar a minha vez e me controlar, expressar frustrações adequadamente, não deixar se intimidar, nomear sentimentos e empatia, expressar direitos e necessidades adequadamente, participar de temas e discussões, elogiar, beijar e abraçar, fazer e atender pedidos e agradecer, admitir erros, pedir desculpas e ouvir críticas.

As sessões davam exemplos de comportamentos habilidosos e não habilidosos, relacionando sempre com o contexto a qual cada um está inserido (família, escola, amigos), instigando todos a praticarem as habilidades aprendidas em contexto terapêutico em suas interações. No início de cada encontro, retomava-se o conteúdo do encontro anterior e questionava-se sobre a aplicação das habilidades aprendidas em seus contextos. Foram realizadas também diversas atividades práticas nas quais cada participante representava contextos relacionados ao tema da sessão, sendo proposto a eles que contassem como agiam nessas situações. Posterior a isso, o grupo analisava os comportamentos habilidosos e não habilidosos sugerindo novas formas de se portar perante as situações.

Além disso, questionava-se aos participantes sobre o que aprenderam, do que mais gostaram e do que menos gostaram, incentivando as crianças a se comportarem em todos os contextos que estão inseridos com base nos aspectos apreendidos nas sessões. 
Tabela 2

Sequência das atividades e as habilidades sociais trabalhadas nas sessões de intervenção

\begin{tabular}{|c|c|c|}
\hline Sessões & Tema de cada sessão & $\begin{array}{c}\text { Tempo de } \\
\text { Sessão }\end{array}$ \\
\hline \multicolumn{3}{|c|}{ Coleta de dados Pré-teste com responsáveis legais. } \\
\hline 1 & Cumprimentar e iniciar conversas de civilidade. & 1h30min. \\
\hline 2 & Agradecer, falar coisas boas e expressar opiniões. & 1h30min. \\
\hline 3 & Fazer amigos e ajudar. Brincar e dividir as coisas. & 1h30min. \\
\hline 4 & Esperar a minha vez e me controlar. & 1h30min. \\
\hline 5 & Expressar frustrações adequadamente, não deixar se intimidar. & 1h30min. \\
\hline 6 & Nomear sentimentos e empatia. & 1h30min. \\
\hline 7 & $\begin{array}{l}\text { Expressar direitos e necessidades adequadamente; participar de } \\
\text { temas e discussões. }\end{array}$ & 1h30min. \\
\hline 8 & Elogiar, beijar e abraçar. & 1h30min. \\
\hline 9 & Fazer e atender a pedidos e agradecer. & 1h30min. \\
\hline 10 & Admitir erros, pedir desculpas e ouvir críticas. & 1h30min. \\
\hline
\end{tabular}

Coleta de dados Pós-teste com responsáveis legais.

Nota. Fonte: Recuperado de "Promove - crianças: treinamento de habilidades sociais: promovendo melhores interações sociais e prevenindo problemas de comportamento", de A. T. Bolsoni-Silva., \& A. P. Falcão, 2016, p. 49.

\section{Análise de dados}

Os dados coletados foram sistematizados utilizando-se o software Statistical Package for the Social Sciences (SPSS, versão 20.0, 2011). As análises foram geradas por meio de medidas descritivas (média, mediana, percentil, porcentagem) e foram obtidas as médias dos instrumentos avaliados. Os resultados encontrados por meio da amostragem foram utilizados para fazer a observação entre os resultados do Pré-teste para o Pós-teste nas mensurações dos instrumentos.

\section{Resultados}

\section{Estilos Parentais}

A análise quantitativa deu-se a partir das respostas dos responsáveis legais das crianças, em relação às práticas educativas parentais presentes nas famílias, em Pré-teste e Pós-teste. No Pré-teste, conforme a Tabela 3, como resultado da classificação em escala, cinco dos sete participantes apresentaram estilos parentais de risco de acordo com a correção do teste, apresentando em suas respostas ausência de atenção e de afeto; práticas corporais negativas, 
ameaça e chantagem de abandono e humilhação; relaxamento das regras estabelecidas; orientação de punição e reforçamento baseados no humor dos pais e não pela consistência dos atos praticados pelas crianças.

Um dos participantes apresentou estilo parental regular abaixo da média, indicando em suas respostas algumas características semelhantes às citadas no exemplo anterior de estilo parental de risco, incluindo as práticas positivas, que podemos ter como exemplo a atenção e a distribuição de privilégios adequados e condições favoráveis ao desenvolvimento de virtudes. Outro participante apresentou estilos parentais regulares acima da média, ou seja, tem na relação com seus pais práticas educativas positivas, tais como a monitoria positiva e o comportamento moral bem estabelecidos e com intensidades adequadas.

$\mathrm{Na}$ avaliação do Pós-teste, apenas a mensuração de dois participantes (P1 e P5) apresentaram os mesmos resultados do Pré-teste. O participante P1 permaneceu com o Estilo Parental de Risco, no qual, de acordo com a literatura, prevalecem práticas de punição inconsistente, negligência, disciplina relaxada, monitoria negativa e abuso físico. Já o participante P5, manteve seu status em Estilo Parental Regular Abaixo da Média, sendo alcançados resultados importantes no restante dos participantes do estudo. O participante P2 passou da classificação de Estilo Parental Regular Acima da Média no Pré-teste para Estilo Parental Ótimo ao responder novamente o instrumento no Pós-teste. Além disso, três pais dos participantes (P3, P6 e P7) passaram da classificação de Estilo Parental de Risco para a classificação de Estilo Parental Regular Abaixo da Média. Por fim, a posição do P4 foi a alteração mais significativa observada, passando de Estilo Parental de Risco para Estilo Parental Ótimo.

Mainardes (2018) comenta que, sob a perspectiva analítico-comportamental, todo e qualquer comportamento, por mais complexo que seja, observável ou encoberto, pode ser compreendido a partir do estudo das interações entre organismo e ambiente, organismo e eventos ambientais interagem a todo instante produzindo alterações recíprocas.

Tabela 3

\section{Estilo Parental (IEP) de cada participante no pré-teste e pós-teste}

\begin{tabular}{l|l|l}
\hline Participantes & \multicolumn{1}{|c|}{ IEP Pré-Teste } & \multicolumn{1}{c}{ IEP Pós-Teste } \\
\hline P1 & Estilo Parental de Risco (4) & Estilo Parental de Risco (4) \\
\hline P2 & Estilo Parental Regular Acima da Média (2) & Estilo Parental Ótimo (1) \\
\hline P3 & Estilo Parental de Risco (4) & Estilo Parental Regular Acima da Média (2) \\
\hline P4 & Estilo Parental de Risco (4) & Estilo Parental Ótimo (1) \\
\hline P5 & Estilo Parental Regular Abaixo da Média (3) & Estilo Parental Regular Abaixo da Média (3) \\
\hline P6 & Estilo Parental de Risco (4) & Estilo Parental Regular Abaixo da Média (3) \\
\hline P7 & Estilo Parental de Risco (4) & Estilo Parental Regular Abaixo da Média (3) \\
\hline Médias & Estilo Parental de Risco (3,57) & Estilo Parental Regular Abaixo da Média (2,42) \\
\hline
\end{tabular}

A partir da aplicação do estudo, em que foram trabalhadas diversas maneiras de portarse em situações de conflitos, além da capacidade de admitir erros, expressar frustrações, 
nomear sentimentos, dentre outras habilidades, foi possível observar que as crianças apresentaram melhoras nas relações de práticas familiares, podendo estas terem sido influenciadas indiretamente, pois, durante a aplicação do estudo grupal, não foi trabalhado diretamente com os pais e/ou cuidadores. Todavia, considerou-se que as diferentes estratégias utilizadas por eles são práticas imprescindíveis para o desenvolvimento do repertório comportamental das crianças que abrange tanto comportamentos habilidosos quanto comportamentos não habilidosos.

\section{Problemas de Comportamento}

Para a análise dos resultados, criou-se um banco de dados no programa SPSS, realizando análises de comparação entre escores Pré-teste e Pós-teste por meio do teste CBCL. De acordo com a Tabela 4, o resultado da variável de Isolamento apresentou a mesma média no Préteste e Pós-teste dos participantes P1, P3 e P7 e diminuição em três pontos na média dos participantes P4, P5 e P6. Somente o participante P2 não apresentou melhora nem mesmo permanência no mesmo estado de Isolamento, passando de 15 para 16. Com relação às Queixas somáticas, os participantes P1, P4 e P7 apresentaram a mesma média, porém, os participantes P2, P3 e P6 diminuíram suas médias em quatro, cinco e três, respectivamente. Já o participante P5 apresentou um aumento em três pontos em suas queixas somáticas. Ao observar a variável de Ansiedade e Depressão, verificou-se que houve uma melhora de dois pontos nos participantes (P2, P6 e P7) em seu resultado final. Contudo, os participantes (P1, P3 e P5) apresentaram melhora em um, três e cinco pontos em sua média. Já o participante P4, apresentou um aumento na Ansiedade e Depressão.

Sobre os Problemas Sociais, identificou-se que houve uma melhora nos participantes (P2, P3 e P5) em um ponto. Logo, os participantes P1 e P7 apresentaram a mesma média, ou seja, 11 e oito. Os participantes $\mathrm{P} 4$ e $\mathrm{P} 6$, porém, tiveram um aumento em um ponto em relação às dificuldades sociais. A maioria dos participantes expressou a mesma média em relação aos Problemas de Pensamento no Pré-teste e Pós-teste, sendo eles P1, com oito pontos, P2, com sete pontos, $\mathrm{P} 3$, com 11 pontos, $\mathrm{P} 4$, com sete pontos, e P7, com sete pontos. Somente os participantes P5 e P6 apresentaram melhoras, sendo um e quatro pontos, respectivamente. No tocante à variável de Problemas de Atenção, evidenciou-se que os resultados dos participantes P1, P3 e P6 permaneceram o mesmo no Pré e Pós-teste, sendo eles 19, 18 e 29. Contudo, os participantes P2, P4, P5 e P7 apresentaram melhoras relacionadas aos Problemas de Atenção em três, quatro, dois e dois pontos, respectivamente.

Os Comportamentos Delinquentes dos participantes P1 e P2 permaneceram na mesma pontuação, 16 e 14. Todavia, houve uma melhora nos comportamentos dos participantes, em que o P4 diminuiu 15 pontos, P5 diminuiu três pontos, P6 diminuiu um ponto e P7 dois pontos. Apenas o participante P3 aumentou sua pontuação em um ponto relação aos seus comportamentos delinquentes. A respeito dos Comportamentos Agressivos, constatouse que apenas um participante, P1, manteve-se com a mesma variável, ou seja, 34 pontos, tanto no Pré-teste quanto no Pós-teste. Além disso, observou-se que os demais participantes, 
(P2, P3, P4, P5, P6 e P7) apresentaram melhora significativa no resultado do Pós-teste ao ser comparado com o Pré-teste, sendo essas melhoras de dois, um, 17, dois, dois e quatro pontos.

Sendo assim, as médias gerais foram identificadas no escore fatorial de isolamento 16 no Pré-teste e 12 no Pós-teste, queixas somáticas 12 no Pré-teste e nove no Pós-teste, ansiedade por depressão 22 no Pré-teste e 17 no Pós-teste, problemas sociais 11 no Pré-teste e 10 no Pós-teste, problemas de pensamento nove no Pré-teste e sete no Pós-teste, Problemas de atenção 20 no Pré-teste e 16 no Pós-teste, comportamento delinquente 19 no Pré-teste e 13 no Pós-teste, comportamento agressivo 36 no Pré-teste e 26 no Pós-teste, e, por fim, problemas relacionados ao sexo, 7,5 no Pré-teste e cinco no Pós-teste.

De forma geral, as variáveis que obtiveram melhor resultado para o desenvolvimento social foram ansiedade e depressão, problemas de atenção, comportamento delinquente e comportamento agressivo.

Tabela 4

Child Behavior Checklist (CBCL) de cada participante no pré-teste e pós-teste.

\begin{tabular}{|c|c|c|c|c|c|c|c|c|c|c|c|c|c|c|c|c|}
\hline \multirow{2}{*}{ 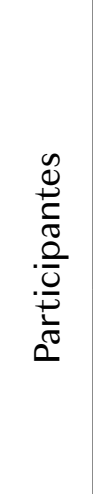 } & \multicolumn{2}{|c|}{$\begin{array}{l}\stackrel{0}{\overparen{T}} \\
\stackrel{0}{0} \\
\frac{\tilde{\pi}}{0} \\
\stackrel{0}{0}\end{array}$} & \multicolumn{2}{|c|}{ 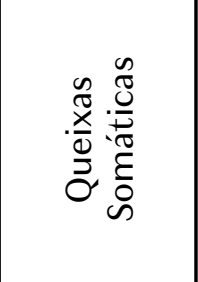 } & \multicolumn{2}{|c|}{ 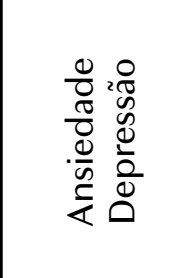 } & \multicolumn{2}{|c|}{ 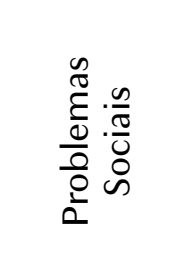 } & \multicolumn{2}{|c|}{ 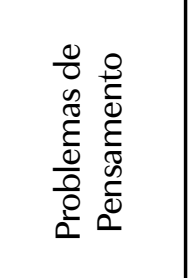 } & \multicolumn{2}{|c|}{ 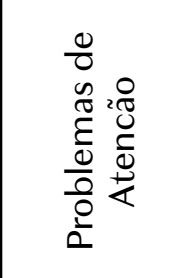 } & \multicolumn{2}{|c|}{ 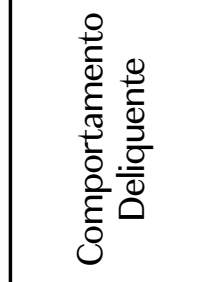 } & \multicolumn{2}{|c|}{ 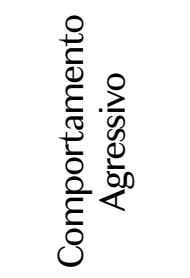 } \\
\hline & 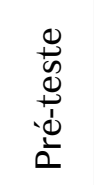 & $\begin{array}{l}\stackrel{0}{0} \\
\stackrel{0}{0} \\
\stackrel{ \pm}{1} \\
\ddot{0} \\
0\end{array}$ & 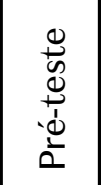 & $\begin{array}{l}\stackrel{0}{\mathscr{y}} \\
\stackrel{0}{0} \\
\stackrel{1}{1} \\
\ddot{0} \\
0\end{array}$ & 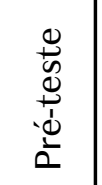 & 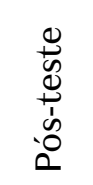 & 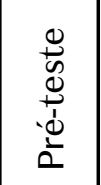 & 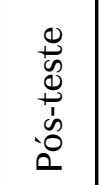 & 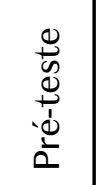 & $\begin{array}{l}\stackrel{0}{0} \\
\stackrel{0}{0} \\
\stackrel{u}{1} \\
\ddot{0} \\
a\end{array}$ & 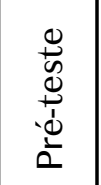 & 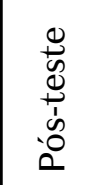 & 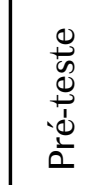 & $\begin{array}{l}\frac{0}{\tilde{y}} \\
\stackrel{0}{\dot{b}} \\
\dot{0} \\
0\end{array}$ & 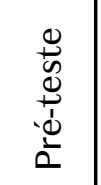 & 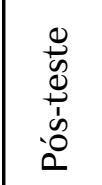 \\
\hline $\mathrm{P} 1$ & 17 & 17 & 11 & 11 & 23 & $22^{\star}$ & 11 & 11 & 8 & 8 & 19 & 19 & 16 & 16 & 34 & 34 \\
\hline $\mathrm{P} 2$ & 15 & 16 & 16 & $12^{\star}$ & 23 & $21^{*}$ & 10 & $9^{*}$ & 7 & 7 & 16 & $13^{*}$ & 14 & 14 & 29 & $27^{*}$ \\
\hline P3 & 16 & 16 & 15 & $10^{\star}$ & 19 & $16^{*}$ & 11 & $10^{\star}$ & 11 & 11 & 18 & 18 & 14 & 15 & 29 & $28^{\star}$ \\
\hline $\mathrm{P} 4$ & 18 & $15^{\star}$ & 9 & 9 & 20 & 27 & 10 & 11 & 7 & 7 & 21 & $17^{\star}$ & 33 & $18^{\star}$ & 46 & $29^{\star}$ \\
\hline P5 & 12 & $99^{\star}$ & 9 & 12 & 24 & $19^{\star}$ & 14 & $13^{\star}$ & 10 & $99^{\star}$ & 22 & $20^{*}$ & 18 & $15^{\star}$ & 32 & $30^{\star}$ \\
\hline $\mathrm{P} 6$ & 20 & $17^{\star}$ & 13 & $10^{\star}$ & 26 & $24^{\star}$ & 23 & 24 & \begin{tabular}{|l|}
13 \\
\end{tabular} & $99^{\star}$ & 29 & 29 & 18 & $17^{\star}$ & 43 & 41 \\
\hline 2 & 9 & 9 & 10 & 10 & 16 & $14^{\star}$ & 8 & 8 & 7 & 7 & 17 & $15^{\star}$ & 19 & $15^{\star}$ & 29 & $25^{\star}$ \\
\hline \multirow{2}{*}{$\frac{\frac{\pi}{\bar{O}}}{\stackrel{0}{\Sigma}}$} & M:16 & $\mathrm{M}: 12^{\prime}$ & M:12 & $\mathrm{M}: 9^{\prime}$ & $\mathrm{M}: 22$ & M:17 & $\mathrm{M}: 11$ & $\mathrm{M}: 10$ & M:9 & $\mathrm{M}: 7^{\prime}$ & M:20 & M:16 & M:19 & $\mathrm{M}: 13$ & M:36 & $\mathrm{M}: 26^{*}$ \\
\hline & & & & $:(1,1)$ & & DP:4, & P:4,9) & & P. 2 & P:1,4 & (DP:4,3) & (DP:5, & (DP:6,5) & $(\mathrm{DP}: 1,3)$ & (DP:7,0) & (DP:5,3) \\
\hline
\end{tabular}

Nota. O sinal (*) representa os comportamentos considerados como melhoras no Pós-Teste, o sinal (M) representa a média e o sinal (DP) representa desvio padrão.

\section{Discussão}

É imprescindível a utilização de instrumentos para a coleta informações sobre o cliente, auxiliando-o na identificação de hipóteses e no planejamento das intervenções, por isso, 
os instrumentos IEP e CBCL foram fundamentais para auxiliar na mensuração dos efeitos decorrentes da aplicação do protocolo PROMOVE - Crianças de Bolsoni-Silva e Falcão (2016).

Identificaram-se no presente estudo a predominância de práticas parentais educativas negativas, estilos parentais de risco em 71,42\% dos participantes, 14,29\% apresentaram estilo parental regular abaixo do esperado e 14,29\% com estilo parental regular acima do esperado. Entre as práticas parentais de risco está a negligência sendo descrita por práticas de ausência de atenção e de afeto, o abuso físico e psicológico, que podem ser caracterizados pela disciplina mediante práticas corporais negativas, ameaça e chantagem de abandono, bem como humilhação e punição inconsistente. Essas ações descrevem a prática na qual os pais se orientam pelo seu próprio humor para punir ou reforçar a criança, levando pouco em consideração o comportamento praticado pelo filho. A disciplina relaxada, por sua vez, compreende a falta de regras e/ou relaxamento das regras estabelecidas. Por outro lado, a monitoria negativa pode ser descrita como o uso excessivo de instruções e de ordens independentemente de seu cumprimento; tal prática tende a levar a um ambiente estressante e hostil na família (Pazzeto \& Toni, 2018).

Gomide (2010) destaca a importância de mencionar os determinantes do comportamento antissocial, o qual, segundo Gomide (2010), está intrinsecamente ligado às práticas parentais utilizadas pelos cuidadores durante toda a fase de desenvolvimento da criança. Para a autora,

Se os cuidadores utilizarem práticas educativas negativas para controlar o comportamento de seus filhos, tais como abuso físico, negligência, ausência ou rigidez de regras, provavelmente consequências indesejadas serão encontradas no decorrer da infância da criança e mais marcantemente quando entrarem na adolescência (p. 261).

Algumas dificuldades como a apatia, o fracasso escolar e o aparecimento de comportamentos autodestrutivos, como o uso de drogas e comportamentos agressivos ou infratores, podem surgir, afetando o desenvolvimento da criança. Para Gomide (2010, p. 261),

Por outro lado, se os cuidadores utilizarem predominantemente práticas parentais positivas para educar estarão desenvolvendo em seus educandos comportamentos pró-sociais que facilitarão a convivência entre os membros da família, na relação com os pares, no processo de aprendizagem, enfim, nas várias áreas importantes para o desenvolvimento saudável do ser humano.

A aprendizagem de respostas é considerada como problemas de comportamento que seguem os mesmos princípios de todo e qualquer comportamento que é adquirido e mantido em seu repertório comportamental ao longo das interações vivenciadas pelo indivíduo. Consequentemente, crianças inseridas em contextos assertivos responderão de forma sensível aos estímulos aversivos, pois aprenderão com facilidade a se proteger e a enfrentar esses estímulos, apresentando comportamentos de autoproteção; pessoas que 
praticam as virtudes em geral não cometem comportamentos antissociais. Nessa perspectiva, os problemas comportamentais podem ser considerados como categorias de respostas operantes e respondentes, as quais são controladas por contingências de reforço e de punição, adquiridas e mantidas por meio de características funcionais na interação organismo-ambiente (Mainardes, 2018); por isso, é fundamental o papel dos pais e familiares.

Já a aplicação do instrumento de avaliação $\mathrm{CBCL}$ avalia classes de comportamentos, reatividade emocional, ansiedade/depressão, queixas somáticas, problemas de atenção, comportamento agressivo e problemas de pensamento (Gallo, Grossi \& Wielewicki, 2011), permitindo verificar o repertório geral das crianças, assim como os dados quantitativos acerca do comportamento de cada um, comparando-se os dados obtidos antes e depois da intervenção, ou seja, no Pré-teste e Pós-teste. Desse modo, com base em Achenbach e Rescorla (2001), todos os resultados estão acima da média da padronização do instrumento, sinalizando problemas de comportamentos tanto no Pré-teste quanto no Pós-teste. Contudo, observou-se a diminuição no escore do Pós-teste em relação ao Pré-teste. Isso significa que houve melhora nos comportamentos das crianças, mas ainda são necessárias intervenções clínicas em casos específicos.

A maioria apresentou problemas de Isolamento. De acordo com Ferreira et al. (2013), as relações entre pares apresentam-se como fator fundamental no desenvolvimento de crianças e de jovens, sendo que a prevenção é imprescindível para que problemas relacionados ao comportamento deixem de serem indicativos de problemas com consequências de curto e longo prazo, o que parece ter auxiliado nas demandas percebidas nesses aspectos.

Crianças expostas a situações de trauma, como a negligência, podem apresentar manifestações somáticas, como a dor de cabeça, dor de barriga e tensão muscular, entre outros, visto que a somatização são sintomas que não podem ser explicados por causas patológicas nem atribuídos a doenças físicas (Ferreira et al., 2014). Sendo assim, no que diz respeito às queixas somáticas, observou-se que cinco dos sete participantes apresentaram melhoras ao responder à ferramenta no Pós-teste, o que indica efetividade no processo de intervenção.

Em relação às manifestações clínicas mais comuns de ansiedade e depressão em crianças, Bahls (2002) indica que são representadas pelos sintomas físicos, sendo seguidas por ansiedade, fobias, agitação psicomotora ou hiperatividade, irritabilidade, diminuição do apetite com falha em alcançar o peso adequado, e alterações do sono. Cita também, que pode ocorrer, em menor frequência, enurese e encoprese, bem como, fisionomia triste, comunicação deficiente e choro frequente na forma de comportamento agressivo e destrutivo.

Os autores Fu, Curatolo e Friedrichc (2000) apontam que a depressão é a falta de esperança e a sensação de que não é possível realizar uma mudança e isso pode levar as pessoas ao suicídio. Alguns sintomas apresentam-se com frequência, tais como insônia, alterações de apetite e peso, perda de energia, isolamento social e pouca expectativa em relação ao futuro. Nos resultados de ansiedade e de depressão, notou-se melhora em 85,74\% das crianças participantes do presente estudo, sendo que a aplicação do treinamento trouxe relevante progresso, mesmo havendo piora em $14,29 \%$ das crianças participantes. 
Com relação aos Problemas Sociais, Catão (2011) aponta que se referem a produtos de um sistema social, econômico, político e cultural. São fenômenos sociais configurados no jogo dessas relações, nas intersubjetividades em ato durante o viver cotidiano. Ao final do programa, observou-se melhora em 42,87\% dos participantes, sendo $28,58 \%$ permaneceram com o mesmo resultado ao responder o Pós-teste, e um total de $28,58 \%$ apresentaram uma elevação à média, no tocante aos problemas sociais, o que indica que essas crianças apresentam menor dificuldade nas interações sociais. Ressalta-se, porém, que esse é um número baixo em relação ao percentual total, compreendendo que é fundamental a continuação do desenvolvimento das habilidades sociais.

Em 28,58\% dos participantes, notou-se melhora com relação aos problemas de pensamento e 71,45\% apresentaram o mesmo resultado tanto no Pré-teste quanto no Pósteste. Com isso, pôde-se compreender que a maioria dos participantes continua apresentando dificuldades de comportamentos encobertos, sendo imprescindível trabalhar tanto com a identificação dos sentimentos quanto com a expressão deles, pois isso contribuem para o desenvolvimento de habilidades sociais.

Já os resultados dos Problemas de Atenção podem estar relacionados ao Transtorno do Déficit de Atenção com Hiperatividade (TDAH), de modo que precisa ser realizado um diagnóstico diferencial quando houver suspeita e descartar a presença de deficiência intelectual, visto que essa patologia pode causar problemas de atenção, de hiperatividade e de impulsividade (Rohde, Barbosa, Tramontina \& Polanczyk, 2000). Não obstante, 57,16\% dos participantes apresentaram melhora na atenção e 42,87\% apresentaram os mesmos resultados ao responder o Pré-teste e o Pós-teste, indicando que a maioria apresenta menor dificuldade em contextos que demandam de atenção.

De acordo com Mainardes (2018), os problemas de comportamento seguem os mesmos princípios de todo e qualquer comportamento que é adquirido e mantido em seu repertório comportamental ao longo das interações vivenciadas pelo indivíduo. Ao observar os resultados, obtiveram-se melhorias com relação aos comportamentos, sendo que o Comportamento Delinquente teve melhora em $57,16 \%$ dos participantes, mas manteve-se o mesmo resultado em 28,58\%. Já o Comportamento Agressivo, obteve melhora significativa em 85,74\%, permanecendo 14,29\% dos participantes no mesmo resultado em Pré-teste e Pós-teste. Esses resultados indicam que a maioria das crianças ampliou favoravelmente comportamentos em seus repertórios, com o número de sessões; contudo, é fundamental que seja trabalhado o autoconhecimento voltado à análise de comportamentos habilidosos $\mathrm{e}$ não habilidosos, apresentados em contextos familiares e sociais.

Assim, podemos notar que a ampliação do repertório comportamental das crianças, com base no programa PROMOVE - Crianças de Bolsoni-Silva e Falcão (2016), desenvolvendo habilidades sociais, possibilita a elas uma melhor interação da criança com o ambiente. Segundo Del Prette e Del Prette (2017), as habilidades sociais são aprendidas, e dessa maneira, podem ser ensinadas de modo incidental ou de modo planejado, por meio de ensino ou terapias individuais ou grupo terapias. 


\section{CONSIDERAÇÕES FINAIS}

A aplicação do programa PROMOVE - Crianças de Bolsoni-Silva e Falcão (2016) demonstrou relevância ao se estudar e se aplicar projetos que desenvolvam as habilidades sociais, haja vista que possibilita a ampliação do repertório comportamental das crianças e adolescentes, permitindo o treino de novos comportamentos. Pôde-se verificar melhora nos comportamentos mensurados por meio do treinamento de habilidades sociais e da ampliação no repertório comportamental dos participantes.

Uma importante evidência da efetividade do programa desenvolvido foi obtida por meio do uso de diferentes medidas de avaliação, as quais possibilitaram a avaliação e a percepção dos pais frente aos seus respectivos filhos por meio do $C B C L$ e as relações familiares mediante o IEP. Os instrumentos apontaram melhoras nos comportamentos das crianças e evidenciaram consistentemente o aumento de comportamentos pró-sociais presentes em seus repertórios adquiridos durante o processo interventivo.

O trabalho com grupos mostrou-se uma alternativa viável para a demanda apresentada na clínica-escola. Notou-se a necessidade da continuidade do treinamento grupal, sendo o número de sessões limitante para se ter um bom desempenho e domínio de comportamentos habilidosos. Denota-se, entretanto, a limitação sobre demandas clínicas e não clínicas de parte das crianças que mantiveram escores mais baixos, sendo esses encaminhados para intervenções psicoterapêuticas individuais.

É possível concluir que o âmbito familiar pode promover comportamentos socialmente adequados, assim como favorecer o surgimento e/ou manutenção de comportamentos inadequados. Por isso, identificou-se também a importância de trabalhar não somente com as crianças, mas também com os pais e/ou cuidadores, pois a maior parte das interações que as crianças vivem é no contexto familiar, aumentando assim a efetividade do processo interventivo.

Com isso, compreender a história de vida dos participantes, o contexto social e cultural que eles estão inseridos e compreender ou entender melhor os problemas de comportamento, assim como estilos parentais prejudiciais ao desenvolvimento, são aspectos fundamentais para que se possa realizar uma intervenção eficaz, de prevenção. Sendo assim, o tratamento precoce auxiliará as crianças a se desenvolverem com qualidade de vida evitando prejuízos ao desenvolvimento e favorecendo a elaboração de vivências relacionadas às habilidades sociais.

Em suma, ressalta-se a imprescindibilidade de pesquisas científicas que objetivem a compreensão, bem como o desenvolvimento e o domínio das práticas educativas positivas e comportamentos habilidosos, proporcionando melhor qualidade de vida e nas interações sociais. 


\section{REFERÊNCIAS}

Achenbach, T. M., \& Rescorla, L. A. (2001). Manual para Formulários e Perfis da Escola ASEBA. Burlington: Universidade de Vermont, Centro de Pesquisa para Crianças, Jovens e Famílias.

Bahls, S.-C. (2002.) Aspectos clínicos da depressão em crianças e adolescentes. Jornal de Pediatria, 78(5), 359-366.

Bee, H., \& Boyd, D. (2011). A Criança em Desenvolvimento (12a ed.). Porto Alegre: Artmed.

Bolsoni-Silva, A. T. (2002). Habilidades sociais: breve análise da teoria e dá prática à luz da análise do comportamento. Bauru, 6(2), 233-242.

Bolsoni-Silva, A. T., \& Falcão, A. P. (2016). Promove - crianças: treinamento de habilidades sociais: promovendo melhores interações sociais e prevenindo problemas de comportamento. São Paulo: CETEPP.

Catão, M. F. (2011). O ser humano e problemas sociais: questões de intervenção. Temas em Psicologia, 19(2), 459-465.

Cia, F., \& Barham, E. J. (2009). Repertório de habilidades sociais, problemas de comportamento, autoconceito e desempenho acadêmico de crianças no início da escolarização. Estudos em Psicologia, 26(1), 45-55. Recuperado de https://www.scielo.br/ pdf/estpsi/v26n1/a05v26n1.pdf

Del Prette, Z. A. P., \& Del Prette, A. (1996). Habilidades sociais: Uma área em desenvolvimento. Psicologia Reflexão e Crítica, 9(2), 233-255.

Del Prette, Z. A. P., \& Del Prette, A. (2008). Psicologia das relações interpessoais: vivências para um trabalho em grupo (9a ed.). Petrópolis: Vozes.

Del Prette, Z. A. P., \& Del Prette, A. (2017). Habilidades sociais: intervenções efetivas em grupos (2a ed.). São Paulo: Pearson.

Farias, A. K. C. R., Fonseca. F, N, \& Nery, L, B. (2018). Teoria e Formulação de Casos em Análise Comportamental Clínica. Artmed: Porto Alegre.

Ferraz, M. R. P. (2005). A Terapia Comportamental Infantil em Grupo e sua Aplicação nos Transtornos de Aprendizagem. In H. J. Guilhardi, \& N. C. Aguirre (Orgs.), Sobre comportamento e cognição: Expondo a variabilidade (pp. 386-399). Santo André: ESETec.

Ferreira, D., Santos, A. J., Ribeiro, O., Freitas, M., Correia, J. V., \& Rubin, K. (2013). Isolamento social e sentimento de solidão em jovens adolescentes. Análise Psicológica, 31(2), 117-127.

Ferreira, S., Martins, M. J., Monteiro, S., \& Pereira, A. (2014). Inventário de Somatização para Crianças. Psicologia, Saúde \& Doenças, 15(3), 751-767.

Fu, L. I., Curatolo, E., \& Friendrichc, S. (2000). Transtornos afetivos. Revista Brasileira Psiquiatria, São Paulo, pp. 24-27.

Gallo, A., Grossi, R., \& Wielewicki, A. (2011). Instrumentos na prática clínica: CBCL como facilitador da análise funcional e do planejamento da intervenção. Temas em Psicologia, 19(2), 513-523. 
Gomide, P. I. C. (2010). Comportamento moral: uma proposta para o desenvolvimento das virtudes. Curitiba: Juruá.

Gomide, P. I. C. (2014). Inventário de estilos parentais: modelo teórico, manual de aplicação, apuração e interpretação. Petrópolis: Vozes.

IBM SPSS. Statistics for Windows, Versão 20.0. Nova York: IBM Corp.

Salvo, C. G., Mazzarotto I. H. K., \& Löhr, S. S. (2005). Estimulando o desenvolvimento de habilidades sociais em idade escolar. Revista Brasileira Crescimento Desenvolvimento Humano, 15(1), 46-55.

Mainardes, L. W. P. (2018). Estilos parentais e risco de problemas de comportamento em crianças inseridas em um serviço de proteção básica do Sistema Único de Assistência Social (Dissertação de Mestrado). Universidade Estadual de Londrina, Londrina-PR, Brasil.

Papalia, D. E. (2013). Desenvolvimento humano (12a ed.). Porto Alegre: AMGH.

Pazzetto, T., \& Toni, C. G. S. (2018). Grupo de orientação a pais em clínica-escola de psicologia. Revista PsicoFAE: Pluralidades em Saúde Mental, 7(2), 69-86.

Portella, M. (2011). Estratégias de THS: treinamento em habilidades sociais. Rio de Janeiro: CPAF.

Rohde, L. A.; Barbosa, G., Tramontina, S. \& Polanczyk, G. (2000). Transtorno de déficit de atenção/hiperatividade. Revista Brasileira: Psiquiatria, 22(2), 7-11.

Weber, L. (2006). Família e Desenvolvimento, Visões Interdisciplinares. Curitiba: Juruá.

Recebido em: 26-11-2019

Primeira decisão editorial: $30-04-2020$

Aceito em: 14-05-2020 